\title{
Covariant description of isothermic surfaces
}

\author{
J. Tafel \\ Institute of Theoretical Physics, University of Warsaw, \\ Hoża 69, 00-681 Warsaw, Poland, email: tafel@fuw.edu.pl
}

\begin{abstract}
We present a covariant formulation of the Gauss-Weingarten equations and the Gauss-Mainardi-Codazzi equations for surfaces in 3-dimensional curved spaces. We derive a coordinate invariant condition on the first and second fundamental form which is locally necessary and sufficient for the surface to be isothermic. We show how to construct isothermic coordinates.
\end{abstract}

Keywords: isothermic surfaces, the Gauss-Weingarten equations

MCS: 53C42, 53A35

\section{Introduction}

Studies of isothermic surfaces were originated in 1837 by Lame [1] who considered surfaces of constant temperature in a solid body. Bertrand [2] is an author of the present definition of isothermic surfaces as surfaces admitting coordinates in which the first fundamental form is conformally flat and the second one is diagonal. Their basic properties were found by Bour [3], Darboux [4], Calapso [5] and Bianchi [6] (see [7] for a review). A transformation given by Darboux and developed by Bianchi allow to obtain a family of new isothermic surfaces from a given one. This property was crucial for an interpretation of isothermic surfaces as soliton surfaces by Cieśliński, Goldstein and Sym [8]. They recognized the GaussMainardi-Codazzi equations for the isothermic surfaces as a completely integrable system. These results initiated a new interest in isothermic surfaces (see [9-11] for a review), including higher dimensions [12,13], discretizations [14] and their generalizations (see e.g. [15]).

Most studies on isothermic surfaces refer to surfaces in a flat or conformally flat 3-dimensional space (see [16] and references therein for surfaces in symmetric spaces). Moreover, their definition is coordinate dependent. The main result of this paper is a coordinate invariant characteristic of isothermic surfaces which is also valid in a general curved 3-dimensional space.

In section 2 we give a covariant formulation of the generalized Gauss-Weingarten (GW) equations and their integrability conditions known as the Gauss-MainardiCodazzi (GMC) equations. We also present a method of a construction of a surface 
in flat space $R^{3}$ with prescribed fundamental forms, which is simpler than passing through the full system of the GW equations.

In section 3 we analyze equations for a coordinate transformation which put a 2-dimensional Riemannian metric $g_{a b}$ into a conformally flat form and, simultaneously, diagonalizes a symmetric tensor $K_{a b}$. Their integrability condition reads $d \omega=0$, where $\omega$ is a differential form defined by the metric and the tensor. This equation, with $g_{a b}$ and $K_{a b}$ corresponding to fundamental forms $g_{I}$ and $g_{I I}$ of a surface, should complete the GW and the GMC equations in the case of isothermic surfaces. It is conformally invariant and it allows an easy verification, in any system of coordinates, if a surface corresponding to some $g_{I}$ and $g_{I I}$ is isothermic. If it is satisfied a construction of isothermic coordinates can be performed in quadratures.

If we represent a surface as a graph of the form $x^{3}=f\left(x^{1}, x^{2}\right)$ and express $g_{I}$ and $g_{I I}$ in terms of $f$, then condition $d \omega=0$ becomes a fourth order nonlinear equation for the function $f$. In principle, this equation can be used to find isothermic surfaces in a Riemannan 3-space.

\section{The Gauss-Weingarten equations}

Let $S$ with coordinates $\xi^{a}$, where $a \in\{1,2\}$, be a 2-dimensional surface in a 3-dimensional manifold $M$ with metric $\tilde{g}$ and coordinates $x^{i}, i \in\{1,2,3\}$. The induced metric (the first fundamental form) is given by

$$
g_{I}=g_{a b} d \xi^{a} d \xi^{b}, g_{a b}=\tilde{g}_{i j} x_{, a}^{i} x_{, b}^{j}
$$

where

$$
\frac{\partial x^{i}}{\partial \xi^{a}}=x_{, a}^{i} .
$$

Vectors $t_{a}=x_{, a}^{i} \partial_{i}$ are tangent to $S$ and orthogonal to the unit vector $n=n^{i} \partial_{i}$

$$
n_{i} x_{, a}^{i}=0, n_{i} n^{i}=1 .
$$

The field $x_{, a}^{i}$ transforms as a vector under transformations of coordinates $x^{i}$ and as a covector under a change of $\xi^{a}$. Thus, it makes sense to define its covariant derivative on $S$ as

$$
D x_{, a}^{i}=d x_{, a}^{i}+\tilde{\omega}_{j}^{i} x_{, a}^{j}-\omega_{a}^{b} x_{, b}^{i},
$$

where $\tilde{\omega}_{j}^{i}$ and $\omega_{b}^{a}$ are the Levi-Civita connections related, respectively, to metric $\tilde{g}$ and $g_{I}$. The tensorial 1-form $D x_{, a}^{i}$ transforms as $x_{, a}^{i}$ under a change of coordinates. Its decomposition in the basis $d \xi^{a}$ yields

$$
D x_{, a}^{i}=x_{a b}^{i} d \xi^{b},
$$


where

$$
x_{a b}^{i}=x_{, a b}^{i}+\tilde{\Gamma}_{j k}^{i} x_{, a}^{j} x_{, b}^{k}-\Gamma_{a b}^{c} x_{, c}^{i}
$$

and $\tilde{\Gamma}^{i}{ }_{j k}$ and $\Gamma_{a b}^{c}$ are, respectively, the Christoffel symbols of $\tilde{g}$ and $g_{I}$. Note that $x_{a b}^{i}$ is symmetric in the lower indices.

For any pair $(a, b)$ we can decompose vector $x_{a b}^{i} \partial_{i}$ in the basis $\left\{t_{a}, n\right\}$

$$
x_{a b}^{i}=x_{, d}^{i} f_{a b}^{d}+n^{i} f_{a b} .
$$

Unknown coefficients $f_{a b}$ and $f_{a b}^{c}$ have to be symmetric in indices $(a, b)$. The contraction of (7) with $n_{i}$ yields $f_{a b}=-K_{a b}$, where $K_{a b}$ are components of the second fundamental form (the exterior curvature) of $S$ given by

$$
g_{I I}=K_{a b} d \xi^{a} d \xi^{b}=D n_{i} d x^{i}=\left(n_{i, j}-\tilde{\Gamma}_{i j}^{k} n_{k}\right) x_{, a}^{j} x_{, b}^{i} d \xi^{a} d \xi^{b} .
$$

A contraction of (7) with $\tilde{g}_{i j} x_{, c}^{j}$ shows that $f_{a b c}=-f_{b a c}$. This together with $f_{a b c}=f_{a c b}$ yields $f_{a b c}=0$. Hence,

$$
D x_{, a}^{i}=-n^{i} K_{a b} d \xi^{b}
$$

Equation (9) is a part of the Gauss-Weingarten equations [10]. In order to complete them we calculate the covariant derivative of $n^{i}$ written in the form

$$
n^{i}=\frac{1}{2} \tilde{\eta}_{j k}^{i} x_{, a}^{j} x_{, b}^{k} \eta^{a b}
$$

Here $\tilde{\eta}^{i j k}$ and $\eta^{a b}$ are the Levi-Civita completely antisymmetric tensors corresponding to $\tilde{g}$ and $g_{I}$, respectively. Taking into account (9) and $D \eta^{i j k}=D \eta^{a b}=0$ one obtains

$$
D n^{i}=x_{, a}^{i} K_{b}^{a} d \xi^{b} .
$$

Equations (9) and (11) generalize the GW equations. Note that for a nonvanishing Gauss curvature $K=\operatorname{det} K_{a}^{b} \neq 0$ condition (11) together with $n^{2}=1, K_{[a b]}=$ 0 and $g_{a b}=x_{, a}^{i} \tilde{g}_{i j} x_{, b}^{j}$ implies $n_{i} x_{, a}^{i}=0$ and (9). To show this one can take contractions of (11) with $n_{i}$ and $g_{i j} x_{, c}^{j}$ and repeat the proof of $f_{a b c}=0$ following (7).

Assume that a 3-dimensional metric $\tilde{g}_{i j}$ on $M$ and tensors $g_{a b}$ and $K_{a b}$ on a 2-dimensional manifold $S$ are given. If there exist solutions $x^{i}\left(\xi^{a}\right)$ and $n^{i}\left(\xi^{a}\right)$ of equations (9), (11) and constraints (11) and (3), then $S$ can be immersed in $M$ in such a way that $g_{a b}$ and $K_{a b}$ are, respectively, the first and the second fundamental form of $S$. Let us consider equations (2), (9) and (11) as an overdetermined system for $x^{i}, x_{, a}^{i}$ and $n^{i}$. Covariant derivatives of constraints (11) and (3) vanish, hence, it is sufficient to assume them in a single point. Integrability of (2) is assured by the symmetry $x_{a b}^{i}=x_{b a}^{i}$ following from (9). Integrability conditions of (9) 
and (11) yield the generalized GMC equations. They coincide with the following equations obtained within the Arnowitt-Deser-Misner decomposition [17] of the Einstein tensor of $\tilde{g}$

$$
\begin{gathered}
\tilde{G}_{i j} n^{i} n^{j}=\frac{1}{2}\left(H^{2}-K_{a b} K^{a b}-R\right) \\
\tilde{G}_{i j} n^{i} x_{, a}^{j}=\left(H \delta_{a}^{b}-K_{a}^{b}\right)_{\mid b} .
\end{gathered}
$$

Here $\tilde{G}_{i j}$ is the Einstein tensor of $\tilde{g}, H=K_{a}^{a}$ is the mean curvature of $S, R$ is the Ricci scalar of $g_{I}$ and ${ }_{\mid b}$ denotes the covariant derivative corresponding to $g_{I}$. Note that for a 2-dimensional surface $\frac{1}{2}\left(H^{2}-K_{a b} K^{a b}\right)$ is just the Gaussian curvature $K$.

For general $\tilde{g}$ equations (12) and (13) are additional constraints for $x^{i}, x_{, a}^{i}$ and $n^{i}$. They can be further differentiated until one obtains conditions which contain only components of fundamental forms $g_{I}$ and $g_{I I}$. Equations (12) and (13) immediately reduce to equations for $g_{I}$ and $g_{I I}$ if $\tilde{g}$ is an Einstein's metric, $\tilde{G}_{i j}=\Lambda \tilde{g}_{i j}$. 3-dimensional metrics of this type are locally equivalent to standard metrics of the flat space $R^{3}$, sphere $S_{3}$ or pseudosphere (hyperbolic space) $H_{3}$. In these cases, given forms $g_{I}$ and $g_{I I}$ which satisfy (12) and (13), one can find a corresponding surface via solving the linear system (9)-(11).

In the case of $R^{3}$ and $K \neq 0$ this procedure can be simplified by using the tensor

$$
g_{I I I}=K_{a c} K_{b}^{c} d \xi^{a} d \xi^{b}=H g_{I I}-K g_{I}
$$

called the third fundamental form. It follows from (11) that

$$
g_{I I I}=\bar{n}_{, a} \bar{n}_{, b} d \xi^{a} d \xi^{b},
$$

where $\bar{n}$ denotes the normal vector in the Cartesian coordinates. In the region, where $n^{3} \neq 1$, equation (15) can be written in the form

$$
g_{I I I}=\frac{d n d n^{*}}{\left(1+\frac{1}{4} n n^{*}\right)^{2}}
$$

where $n=\frac{n^{1}+i n^{2}}{1-n^{3}}$ and $n^{*}$ is its complex conjugate. If $K \neq 0$ then $g_{I I I}$ is nondegenerate, functions $n$ and $n^{*}$ must be independent and the rhs of (16) is the standard spherical metric. An interesting consequence of (14) and (15) is that metric of any minimal $(H=0)$ surface in $R^{3}$ satisfies

$$
g_{I}=|K|^{-1} d \bar{n} d \bar{n}
$$

exactly as it is for constant radius spheres, which are not minimal surfaces. 
Given $g_{I}$ and $g_{I I}$ satisfying the GMC equations, from (16) one can derive the complex stereographic coordinate $n$ and normal vector $\bar{n}$. To this end one should first find complex coordinates $\xi, \xi^{*}$ in which form (14) is conformally flat

$$
g_{I I I}=e^{2 u} d \xi d \xi^{*} .
$$

This can be done by solving the complex linear equation

$$
d \xi \wedge\left(\theta^{1}+i \theta^{2}\right)=0,
$$

where $\theta^{a}$ is an orthonormal basis for tensor $g_{I I I}$. Equation (19) has the form $v(\xi)=0$, where $v$ is a complex vector field. If it is analytic, equation (19) can be solved by the method of characteristics.

It follows from (16) and (18) that $n$ is a holomorphic function of $\xi$ (or antiholomorphic but then we can change this by means of a reflection in $R^{3}$ ) and

$$
e^{-u}=\frac{1}{\sqrt{\dot{n}^{*}}} \frac{1}{\sqrt{\dot{n}}}+\frac{n^{*}}{\sqrt{\dot{n}^{*}}} \frac{n}{4 \sqrt{\dot{n}}},
$$

where $\dot{n}=n_{, \xi}$. Let us consider holomorphic extension of equation (20) to two independent complex variables, still denoted by $\xi$ and $\xi^{*}$, and then fix $\xi^{*}=\xi_{0}^{*}$. It follows that

$$
e^{-u_{0}}=A_{11} \frac{1}{\sqrt{\dot{n}}}+A_{21} \frac{n}{4 \sqrt{\dot{n}}},
$$

where $u_{0}(\xi)=u\left(\xi, \xi_{0}^{*}\right)$ and $A_{11}$ and $A_{21}$ are complex numbers. If we apply the same procedure to the derivative of (20) over $\xi^{*}$ we obtain

$$
\left(e^{-u_{0}}\right)_{, \xi_{0}^{*}}=A_{12} \frac{1}{\sqrt{\dot{n}}}+A_{22} \frac{n}{4 \sqrt{\dot{n}}} .
$$

Matrix $\left(A_{a b}\right)$ is an element of the group $S L(2, C)$. Equations (21) and (22) can be easily solved with respect to $n$ and $\dot{n}$ giving

$$
n=-4 \frac{A_{11} u_{, \xi_{0}^{*}}+A_{12}}{A_{21} u_{, \xi_{0}^{*}}+A_{22}}, \quad \dot{n}=\frac{e^{2 u_{0}}}{\left(A_{21} u_{\xi_{0}^{*}}+A_{22}\right)^{2}} .
$$

Equation (20) cannot define the stereographic coordinate $n$ better than up to the following transformations, with complex parameters $\alpha$ and $\beta$,

$$
n \rightarrow \frac{\alpha n+\beta}{-\beta^{*} n+\alpha^{*}}, \quad|\alpha|^{2}+|\beta|^{2}=1
$$

which correspond to rigid rotations in $R^{3}$. One can use (24) to reduce (23) to

$$
n=-4 A\left(u_{\xi_{0}^{*}}+B\right), \dot{n}=A e^{2 u_{0}}
$$


with a complex constant $B$ and a real positive constant $A$. Substituting (25) back into (20) yields equality

$$
1+4 A^{2}\left|u_{, \xi_{0}^{*}}+B\right|^{2}=A e^{u_{0}+u_{0}^{*}-u},
$$

which should be satisfied for all values of $\xi$. In order to fit constants $A$ and $B$ algebraically it is sufficient to impose (26) at three points. Given them we can calculate the normal vector $\bar{n}$ and substitute it into (11) written as

$$
x_{, a}^{i}=K^{-1}\left(H \delta_{a}^{b}-K_{a}^{b}\right) n_{, b}^{i} .
$$

For complex conjugated $\xi$ and $\xi^{*}$ functions $x^{i}\left(\xi, \xi^{*}\right)$ follow from integration of (27) up to constants, which together with (24) represent an Euclidean motion in $R^{3}$. In this way one obtains a parametric description of the surface with prescribed fundamental forms, provided that they satisfy the GMC equations. This procedure seems to be much simpler than passing through the full system of the GW equations (see, e.g. [18]).

\section{Isothermic surfaces}

The conformal transformation

$$
\tilde{g} \rightarrow \Omega^{2} \tilde{g}
$$

implies

$$
g_{I} \rightarrow \Omega^{2} g_{I}, \quad g_{I I} \rightarrow \Omega g_{I I}+n(\Omega) g_{I} .
$$

Fundamental properties of isothermic surfaces, which have conformally flat $g_{I}$ and diagonal $g_{I I}$ in some coordinates $\xi^{\prime a}$, are preserved by these transformations. In view of this, if $\tilde{g}$ is conformally flat, then in order to find isothermic surfaces one can first look for solutions of the flat GMC equations written in the coordinates $\xi^{\prime a}$. In this case one can reduce the problem to the Calapso equation [9]. This approach fails if $\tilde{g}$ is not conformally flat. For this reason, in this section we will characterize isothermic surfaces independly of the GW and GMC equations. Our aim is to find a coordinate invariant condition which is locally necessary and sufficient for the existence of isothermic coordinates. An interpretation of $K_{a b}$ as the second fundamental form is not relevant for this condition.

Theorem 3.1. Let $g_{a b}$ and $K_{a b}$ be, respectively, a 2-dimensional Riemannian metric and a symmetric tensor, which are locally linearly independent. Then, $g_{a b}$ is conformally flat and $K_{a b}$ is diagonal in some coordinates iff

$$
d \omega=0,
$$


where

$$
\begin{gathered}
\omega=k_{a}^{b} k_{b \mid c}^{c} d \xi^{a}, \\
k_{a b}=\alpha^{-1}\left(K_{a b}-\frac{1}{2} K_{c}^{c} g_{a b}\right)
\end{gathered}
$$

and $\alpha>0$ is a normalization factor such that $k_{a b} k^{a b}=2$.

Proof. Let $\xi^{\prime a}$ be coordinates in which $g_{12}^{\prime}=0, g_{11}^{\prime}=g_{22}^{\prime}$ and $K_{12}^{\prime}=0$. A transformation from general coordinates $\xi^{a}$ to $\xi^{\prime a}$ has to satisfy the conditions

$$
\begin{gathered}
L_{1}^{a} g_{a b} L_{2}^{b}=0 \\
L_{1}^{a} g_{a b} L_{1}^{b}=L_{2}^{a} g_{a b} L_{2}^{b} \\
L^{a}{ }_{1} K_{a b} L_{2}^{b}=0,
\end{gathered}
$$

where $L^{a}{ }_{b}=\frac{\partial \xi^{a}}{\partial \xi^{\prime b}}$. In view of (33) equation (35) can be replaced by

$$
L_{1}^{a} \hat{K}_{a b} L_{2}^{b}=0
$$

where

$$
\hat{K}_{a b}=K_{a b}-\frac{1}{2} K_{c}^{c} g_{a b}
$$

is the traceless part of $K_{a b}$.

Let $-\alpha$ be a point dependent eigenvalue of $\hat{K}_{b}^{a}$. It must be real since any 2-dimensional metric $g_{a b}$ admits conformally flat coordinates and in these coordinates matrix $\hat{K}_{b}^{a}$ is symmetric. Since $\operatorname{det}\left(\hat{K}_{a b}+\alpha g_{a b}\right)=0$ and $\hat{K}_{a b}$ is traceless it can be written in the form

$$
\hat{K}_{a b}=\alpha\left(2 u_{a} u_{b}-g_{a b}\right),
$$

where $u^{a} u_{a}=1$. Note that $\alpha \neq 0$ since $K_{a b}$ is not proportional to $g_{a b}$. Thus, locally there is $\alpha>0$ or $\alpha<0$ depending on our choice of the eigenvector $u$ (note that transformation $u^{a} \rightarrow \eta^{a b} u_{b}, \alpha \rightarrow-\alpha$ preserves (38)). By virtue of (38) equation (35) leads to $L^{a}{ }_{1} u_{a}=0$ (or $L_{2}^{a} u_{a}=0$ but then we can interchange indices 1 and 2). Hence

$$
L_{1}^{a}=\gamma \eta^{a b} u_{b}
$$

where $\gamma$ is a function. Substituting (39) into (33) yields $L_{2}^{a}=\gamma^{\prime} u^{a}$. Equation (34) shows that $\gamma^{\prime 2}=\gamma^{2}$. Thus, equations (33)-(35) are equivalent to (39) and

$$
L_{2}^{a}= \pm \gamma u^{a} .
$$

From (39) and (40) we obtain the following expressions for the matrix $L^{-1}$

$$
\frac{\partial \xi^{\prime 1}}{\partial \xi^{a}}=\lambda \eta_{a b} u^{b}
$$




$$
\frac{\partial \xi^{\prime 2}}{\partial \xi^{a}}= \pm \lambda u_{a}
$$

where $\lambda$ is a nonvanishing function. Conditions (41) and (42), considered as equations for coordinates $\xi^{\prime a}$, are integrable provided

$$
(\ln |\lambda|)_{, a}=u^{b} u_{a \mid b}-u_{\mid b}^{b} u_{a}
$$

Equation (43) can be written in the form

$$
(\ln |\lambda|)_{, a}=-\frac{1}{2} k_{a}^{b} k_{b \mid c}^{c},
$$

where

$$
k_{a b}=2 u_{a} u_{b}-g_{a b} .
$$

It follows from (45) and $u^{2}=1$ that $k_{a b} k^{a b}=2$. Equality (38) implies (32) and

$$
\alpha^{2}=\frac{1}{2} \hat{K}_{a b} \hat{K}^{a b}
$$

Equation (30) is just an integrability condition of (44).

If $g_{a b}$ and $K_{a b}$ are to be fundamental forms of a surface $S$ in a 3-dimensional Riemannian manifold $M$ they should be compatible with the GMC equations. Points, where $\hat{K}_{a b}=0$ are called umbilics. Out of them the normalized tensor $k_{a b}$ can be defined (it can be still prolongated to umbilics, in which $\hat{K}_{a b}$ has a zero of a finite order). It follows from (29) that $k_{a}^{b}$ and $d \omega$ are conformally invariant. Equation (30) is an additional condition for the surface to be isothermic. In contrary to other approaches, for instance via the Hopf differential [19], this condition can be verified in any system of coordinates.

The proof of Theorem 1 indicates how to find isothermic coordinates $\xi^{\prime a}$ if condition (30) is satisfied. We should first find $\lambda$ from (44) and an unit vector $u^{a}$ from

$$
k_{b}^{a} u^{b}=u^{a}
$$

Then equations (41) and (42) can be solved in quadratures. They are integrable thanks to (30). This procedure is much simpler from that presented in [20].

It follows from (45) that $k_{b}^{a}$ is an involutive matrix

$$
k_{b}^{a} k_{c}^{b}=\delta_{c}^{a} .
$$

Substituting (32) and (48) into equation (30) yields

$$
\eta^{a d}\left(\alpha^{-2} \hat{K}_{a}^{b} \hat{K}_{b \mid c}^{c}\right){ }_{\mid d}=0,
$$

Equation (49) is automatically satisfied if $\tilde{G}_{i j}=\Lambda \tilde{g}_{i j}$ and $H=$ const, since then equation (13) yields $\hat{K}_{b \mid c}^{c}=0$. This reflects a known fact that constant mean curvature surfaces in space forms $R^{3}, S_{3}$ or $H_{3}$ are isothermic. 


\section{Summary}

We have been considering surfaces $S$ in a three dimensional curved space $M$ with metric $\tilde{g}$. The Riemannian connections on $M$ and $S$ allow to write the GW equations and the GMC equations in a covariant way (equations (9)-(13)). Using the third fundamental form we have shown how to simplify a construction of a surface in the flat space $R^{3}$ given fundamental forms $g_{I}$ and $g_{I I}$ satisfying the GMC equations.

Starting with a 2-dimensional Riemannian metric $g_{a b}$ and a symmetric tensor $K_{a b}$ we defined a 1 -form $\omega$, which must be closed if there exist coordinates in which metric is conformally flat and $K_{a b}$ is diagonal (Theorem 1). If these tensors correspond to fundamental forms of a surface $S$, condition $d \omega=0$ is locally necessary and sufficient for the surface to be isothermic. This condition is preserved by transformations of coordinates and conformal transformation (28). This is an advantage with respect to standard descriptions of isothermic surfaces. If $d \omega=0$, a construction of isothermic coordinates can be easily performed.

In principle, one can also use equation $d \omega=0$ in order to find isothermic surfaces together with $g_{I}$ and $g_{I I}$. Locally, every surface $S \subset M$ is a graph of a function $f$ of two coordinates. The induced metric and the normal vector of $S$ depend on $f$ and its first derivatives. Hence, the exterior curvature tensor depends on second derivatives of $f$ and equation $d \omega=0$ is a fourth order nonlinear equation for $f$. Unfortunately, this equation is rather complicated even in the flat space $R^{3}$, compared e.g. to the Calapso equation.

\section{References}

[1] G. Lamé: Mémoire sur les surfaces isothermes dans les corps solides homogenes en équlibre de température, J. Math. Pur. Appl. (Liouville J.) 2, 147-183 (1837)

[2] J. Bertrand: Mémoire sur les surfaces isothermes orthogonales, J. Math. Pur. Appl. (Liouville J.) 9, 117-130 (1844)

[3] E. Bour: Théorie de la déformation des surfaces, J. l'École Imperiale Polytech. 19, Cahier 39, 1-48 (1862)

[4] G. Darboux: Sur les surfaces isothermiques, C. R. Acad. Sci. Paris 128, 1299-1305 (1899)

[5] P. Calapso: Sulla superficie a linee di curvature isoterme, Rend. Circ. Mat. Palermo 17, 275-286 (1903) 
[6] L. Bianchi: Ricerche sulle superficie isoterme e sulle deformazione delle quadriche, Ann. Matem. 11, 93-157 (1905)

[7] P. Klimczewski, M. Nieszporski and A. Sym: Luigi Bianchi, Pasquale Calapso and solitons, Rend. Sem. Mat. Messina, Atti del Congresso Internazionale in onore di Pasquale Calapso, Messina 1998, 223-240 (2000)

[8] J. Cieśliński, P Goldstein and A. Sym: Isothermic surfaces in E3 as soliton surfaces, Phys. Lett. A 205, 37-43 (1995)

[9] C. Rogers and W.K. Schief: Bäcklund and Darboux Transformations. Geometry and Modern Applications in Soliton Theory, Cambridge Texts in Applied Mathematics, Cambridge University Press, 2002

[10] U. Hertrich-Jeromin: Introduction to Möbius Differential Geometry, Cambridge University Press, 2003

[11] F.E. Burstall and D.M.J. Calderbank: Conformal submanifold geometry IIII, arXiv: 1006.5700

[12] Martina Brück, Xi Du, Joonsang Park and Chuu-Lian Terng: The submanifold geometries associated to Grassmannian systems, Memoirs of the American Mathematical Society 155, No 735 (2002)

[13] F.E. Burstall: Isothermic surfaces: conformal geometry, Clifford algebras and integrable systems. in: C.-L. Terng (ed.), Integrable systems, Geometry and Topology, AMS/IP Studies in Advanced Math. 36, 1-82 (2006)

[14] A.I. Bobenko and U. Pinkall: Discrete Isothermic Surfaces, J. Reine Angew. Math. 475 , 187-208 (1996)

[15] A. Doliwa, Generalized isothermic lattices: J. Phys. A: Math. Theor. 40 , 12539-12561 (2007)

[16] F. E. Burstall, N. M. Donaldson, F. Pedit and U. Pinkall: Isothermic submanifolds of symmetric $R$-spaces, J. Reine Angew. Math. 660, 191-243 (2011)

[17] N. Straumann, General Relativity (Second Edition): Graduate Text in Physics, Springer, 2013

[18] Z. Kose, M. Toda and E. Aulisa: Solving Bonnet problems to construct families of surfaces, Balk. J. Geom. Appl. 16 , 70-80 (2011)

[19] A. I. Bobenko: Exploring Surfaces through Methods from the Theory of Integrable Systems. Lectures on the Bonnet Problem, 1999, arXiv:math/9909003 
[20] E. Aulisa, M. Toda and Z. Kose: Constructing isothermal curvature line coordinates on surfaces which admit them, Cent. Eur. J. Math. 11, 1982-1993 (2013) 